\title{
STABILITY OF HARMONIC MAPS AND MINIMAL IMMERSIONS
}

\author{
Y. L. PAN $^{1}$ AND Y. B. SHEN
}

\begin{abstract}
It was proved by J. Simons [10] that there does not exist any stable minimal submanifold in the Euclidean sphere $S^{n}$, and P. F. Leung proved that any stable harmonic map from any Riemannian manifold to $S^{n}$, where $n \geqslant 3$, is a constant. In this paper, we generalize their results and indicate that there are many manifolds having such properties as $S^{n}$.
\end{abstract}

A harmonic map is a differential map which is a critical point of the energy functional, and a minimal immersion is a harmonic isometric immersion [3]. There have been some nonexistence theorems of stable harmonic maps $[4,6-8,11,12]$. On the other hand, it was proved by J. Simons [10] that there does not exist any stable minimal submanifold in the Euclidean sphere. The aim of this paper is to generalize these results.

1. Basic formulas and notation. In this section we establish our notation and state some basic formulas. More details can be found in corresponding references. We shall make use of the following convention on the ranges of indices:

$$
\begin{gathered}
0 \leqslant A, B, C, \ldots \leqslant n+p, \quad 1 \leqslant \alpha, \beta, \gamma, \ldots \leqslant n ; \quad n+1 \leqslant \mu, \nu, \ldots \leqslant n+p ; \\
1 \leqslant i, j, k, \ldots \leqslant m ; \quad m+1 \leqslant r, s \leqslant n, \quad \text { if } m<n .
\end{gathered}
$$

1.1 Harmonic maps $[2,3,9]$. Let $M$ and $N$ be Riemannian manifolds of dimensions $m$ and $n$, respectively, and $f: M \rightarrow N$ a smooth map. We choose local fields of orthonormal frames $\left\{e_{i}\right\}$ and $\left\{e_{\alpha}^{\prime}\right\}$ in $M$ and $N$, respectively, and let $\left\{w_{i}\right\}$ and $\left\{w_{\alpha}^{\prime}\right\}$ be the fields of dual frames.

Under the map $f$, we have $f^{*} w_{\alpha}^{\prime}=\sum_{i} a_{\alpha i} w_{i} . E(f)=\frac{1}{2} \int_{M} \sum_{\alpha, i} a_{\alpha i}^{2} * 1$ is called the energy of $f . f$ is harmonic if the tension tensor $\tau=\sum_{\alpha, i} a_{\alpha i i} e_{\alpha}^{\prime}$ vanishes, where $a_{\alpha i j}$ is the covariant derivative of $a_{\alpha i}$.

For any deformation vector along $f, V=\sum_{\alpha} V_{\alpha} e_{\alpha}^{\prime}$, the second variation of the energy is

$$
I_{f}(V, V)=-\int_{M}\left\{\sum_{\alpha} V_{\alpha}\left(\Delta_{M} V_{\alpha}+\sum_{\beta, \gamma, \delta, i} a_{\beta i} a_{\gamma i} V_{\delta} R_{\beta \alpha \gamma \delta}^{\prime}\right)\right\} * 1,
$$

where $\Delta_{M}$ is the Laplacian of $M$ and $R_{\beta \alpha \gamma \delta}^{\prime}$ is the curvature tensor of $N$.

Received by the editors May 30, 1983 and, in revised form, February 17, 1984.

1980 Mathematics Subject Classification. Primary 58E20; Secondary 53C42.

${ }^{1}$ The first-named author is supported by Max-Planck-Institut für Mathematik, Sonderforschungbereich 40 der Universität Bonn. 
If, for any deformation vector $V$ along $f, I_{f}(V, V)$ is nonnegative, then the harmonic map $f$ is said to be stable.

1.2 Minimal immersions $[1,10]$. When $f: M \hookrightarrow N$ is an isometric immersion from $M$ to $N$, then $f$ is minimal if and only if $f$ is harmonic. Let $f$ be a minimal immersion from $M$ to $N$, and $M$ compact without boundary. For any normal deformation vector $U=\sum_{r} u_{r} e_{r}$ of $f(M)$, the second variation of the volume is given by

$$
\tilde{I}_{f}(U, U)=-\int_{M}\left\{\sum_{r} u_{r}\left[\Delta u_{r}+\sum_{s}\left(Q_{r s}+R_{r s}^{\prime}\right) u_{s}\right]\right\} * 1,
$$

where $u_{r i}, u_{r i j}$ are the covariant derivatives of $u_{r}$,

$$
\begin{gathered}
\Delta u_{r}=\sum_{i} u_{r i i}, \\
Q_{r s}=\sum_{i, j} a_{r i j} a_{s i j},
\end{gathered}
$$

and

$$
R_{r s}^{\prime}=\sum_{i} R_{r i s i}^{\prime}
$$

$f$ is said to be stable if $\tilde{I}_{f}(U, U)$ is nonnegative for any normal deformation vector $U$.

1.3 Submanifolds in the Euclidean sphere $S^{n+p}$ [5]. Let $X: N \hookrightarrow S^{n+p} \subset R^{n+p+1}$ be an isometric immersion. We choose a local field of orthonormal frames $e_{0}, e_{1}, \ldots, e_{n}, e_{n+1}, \ldots, e_{n+p}$ in the Euclidean space $R^{n+p+1}$ such that, restricted to $N$, the vectors $e_{1}, \ldots, e_{n}$ are tangent to $N$ and $e_{0}=-X$; here $X$ is the position vector. Then we have

$$
\begin{aligned}
& d X=\sum_{\alpha} w_{\alpha} e_{\alpha}, \\
& d e_{\alpha}=\sum_{\beta} w_{\alpha \beta} e_{\beta}+\sum_{\mu, \beta} B_{\alpha \beta}^{\mu} e_{\mu} w_{\beta}-X w_{\alpha}, \\
& d e_{\mu}=-\sum_{\alpha, \beta} B_{\alpha \beta}^{\mu} w_{\beta} e_{\alpha}+\sum_{\nu} w_{\mu \nu} e_{\nu},
\end{aligned}
$$

and the second fundamental form of $M$ in $S^{n+p}$ is

$$
B=\sum_{\alpha, \beta, \mu} B_{\alpha \beta}^{\mu} w_{\alpha} \otimes w_{\beta} \otimes e_{\mu}
$$

With the notation

$$
B_{e_{\alpha}, e_{\beta}}=B\left(e_{\alpha}, e_{\beta}\right)=\sum_{\mu} B_{\alpha \beta}^{\mu} e_{\mu},
$$

the square of the length of the second fundamental form $B$ can be written as

$$
\|B\|^{2}=\sum_{\alpha, \beta}\left\langle B_{e_{\alpha}, e_{\beta}}, B_{e_{\alpha}, e_{\beta}}\right\rangle=\sum_{\mu, \alpha, \beta}\left(B_{\alpha \beta}^{\mu}\right)^{2},
$$

where $\langle$,$\rangle denotes the inner product in Euclidean space, and the mean curvature of$ $X(M)$ as

$$
H=\operatorname{trace} B=\sum_{\alpha} B_{e_{\alpha}, e_{\alpha}}=\sum_{\alpha, \mu} B_{\alpha \alpha}^{\mu} e_{\mu}
$$


2. Main results. Firstly, we prove

THEOREM 1. Let $N$ be an $n$-dimensional submanifold in $S^{n+p}$ with second fundamental form $B$ and mean curvature $H$ in $S^{n+p}$. When $n>2+\tilde{B}$, there is no nonconstant stable harmonic map from any compact Riemannian manifold $M$ to $N$, where

$$
\tilde{B}=\left\{\sum_{\alpha, \beta}\left(2 \sum_{\gamma}\left\langle B_{e_{\gamma}, e_{\alpha}}, B_{e_{\gamma}, e_{\beta}}\right\rangle-\left\langle H, B_{e_{\alpha}, e_{\beta}}\right\rangle\right)^{2}\right\}^{1 / 2} .
$$

Proof. Suppose that $f$ is a harmonic map from a compact Riemannian manifold $M$ to $N$. Let $L$ be the space of deformation vector fields on $N$ defined by

$$
L=\left\{\left.\Lambda\right|_{N}: \Lambda \text { is any constant vector in } R^{n+p+1}\right\} .
$$

For any $V \in L$, since it is the projection over $N$ of a certain constant vector in $R^{n+p+1}$, we can write

$$
V=\sum_{\alpha} v_{\alpha} e_{\alpha}, \quad v_{\alpha}=\left\langle\Lambda, e_{\alpha}\right\rangle
$$

Noting $\Delta_{M} v_{\alpha}=\sum_{\beta, \gamma, i} v_{\alpha \beta \gamma} a_{\beta i} a_{\gamma i}$, where $v_{\alpha \beta \gamma}$ is the second covariant derivative of $v_{\alpha}$ with respect to the connection of $T N$, and using the Gauss equation and (1.6)-(1.8), we compute

$$
\begin{aligned}
I_{f}(V, V)=\int_{M}\left\{2 \sum_{\alpha, \beta, i} a_{\alpha i} a_{\beta i} v_{\alpha} v_{\beta}-\sum_{\alpha, \beta, \gamma, \mu, i} a_{\beta i} a_{\gamma i} v_{\alpha} v_{\mu} B_{\alpha \beta \gamma}^{\mu}\right. \\
+\sum_{\alpha, \beta, \gamma, \delta, \mu, i} a_{\beta i} a_{\gamma i} v_{\alpha} v_{\gamma} B_{\alpha \beta}^{\mu} B_{\delta \gamma}^{\mu}-\sum_{\alpha, \beta, i} a_{\beta i} a_{\beta i} v_{\alpha} v_{\alpha} \\
\left.\quad+\sum_{\alpha, \beta, \gamma, \delta, \mu, i} a_{\beta i} a_{\gamma i} v_{\alpha} v_{\delta}\left(B_{\beta \delta}^{\mu} B_{\alpha \delta}^{\mu}-B_{\beta \gamma}^{\mu} B_{\alpha \delta}^{\mu}\right)\right\} * 1,
\end{aligned}
$$

where $v_{\mu}=\left\langle\Lambda, e_{\mu}\right\rangle$ and $B_{\alpha \beta \gamma}^{\mu}$ is the covariant derivative of $B_{\alpha \beta}^{\mu}$.

Since $L$ is a vector space of finite dimension, we can take the trace of $I_{f}(V, V)$ over $L$. Using the same technique of taking traces as in $[4,11]$, we obtain

$$
\text { trace } \begin{aligned}
I_{f} & =2(2-n) E(f)+\int_{M} \sum_{\alpha, \beta, i} a_{\alpha i} a_{\beta i}\left(2 \sum_{\gamma}\left\langle B_{e_{\gamma}, e_{\alpha}}, B_{e_{\gamma}, e_{\beta}}\right\rangle-\left\langle H, B_{e_{\alpha}, e_{\beta}}\right\rangle\right) * 1 \\
& \leqslant 2 E(f)(2-n+\tilde{B}) .
\end{aligned}
$$

The proof is complete.

REMARK 1. In the case $S^{n} \hookrightarrow S^{n+1}$, we have $B=0$, and this theorem becomes Leung's result, which was also obtained by C. K. Peng in [8].

Now we turn to the case of minimal immersion. Suppose $N$ is an $n$-dimensional Riemannian manifold in $S^{n+p}$ and $f: M \rightarrow N$ a minimal immersion, where $M$ is an $m$-dimensional Riemannian manifold. In this case we can choose a local orthonormal basis $e_{0}, e_{1}, \ldots, e_{m}, e_{m+1}, \ldots, e_{n}, e_{n+1}, \ldots, e_{n+p}$ such that $e_{1}, \ldots, e_{m}$ are tangent to $M, e_{1}, \ldots, e_{n}$ are tangent to $N$, and $-e_{0}$ is the position vector $X$. From (1.6)-(1.8), 
restricted to $f(M)$, we have

$$
\begin{aligned}
& d X=\sum_{i} w_{i} e_{i}, \\
& d e_{i}=\sum_{j} w_{i j} e_{j}+\sum_{r, j} a_{r i j} w_{j} e_{r}+\sum_{\mu, j} B_{i j}^{\mu} w_{j} e_{\mu}-X w_{i}, \\
& d e_{r}=-\sum_{i, j} a_{r i j} w_{i} e_{j}+\sum_{s} w_{r s} e_{s}+\sum_{\mu, j} B_{r j}^{\mu} w_{j} e_{\mu}, \\
& d e_{\mu}=-\sum_{i, j} B_{i j}^{\mu} w_{i} e_{j}-\sum_{r, j} B_{r j}^{\mu} w_{j} e_{r}+\sum_{\nu} w_{\mu \nu} e_{\nu},
\end{aligned}
$$

where $\sum_{i} a_{r i i}=0$, since $f$ is a minimal immersion.

Let $L$ be the normal deformation vector space of $f(M)$ which consists of the normal space of $f(M)$ in $N$. If $U \in L$ we can write

$$
U=\sum_{r} u_{r} e_{r}, \quad u_{r}=\left\langle\Lambda, e_{r}\right\rangle,
$$

where $\Lambda$ is a constant vector in $R^{n+p+1}$.

Using (2.5), a straightforward computation shows

$$
\begin{aligned}
u_{r i k}= & -\sum_{j} u_{j}\left(a_{r i j k}+\sum_{\mu} B_{r i}^{\mu} B_{j k}^{\mu}\right)+\sum_{\mu}\left(B_{r i k}^{\mu}-\sum_{j} a_{r i j} B_{j k}^{\mu}\right) u_{\mu} \\
& -\sum_{s} u_{s}\left(\sum_{j} a_{r i j} a_{s j k}+\sum_{\mu} B_{r i}^{\mu} B_{s k}^{\mu}\right)-u_{0} a_{r i k},
\end{aligned}
$$

and $B_{\text {rik }}$ is defined by

$$
\sum_{k} B_{r i k} w_{k}=d B_{r i}+\sum_{k} B_{r k} w_{k i}+\sum_{s} B_{s i} w_{s r}+\sum_{\nu} B_{r i}^{\nu} w_{\nu}
$$

and

$$
u_{0}=\left\langle\Lambda, e_{0}\right\rangle, \quad u_{j}=\left\langle\Lambda, e_{j}\right\rangle, \quad u_{\mu}=\left\langle\Lambda, e_{\mu}\right\rangle .
$$

Thus we obtain from (1.2)

$$
\begin{aligned}
\tilde{I}_{f}(U, U)=-\int_{M}\left\{\sum_{i, r, \mu} u_{r} u_{\mu}\left(B_{r i i}^{\mu}-\sum_{j} a_{r i j} B_{j i}^{\mu}\right)-\sum_{i, j} u_{r} u_{j}\left(a_{r j i i}+\sum_{\mu} B_{r i}^{\mu} B_{j i}^{\mu}\right)\right. \\
\left.+\sum_{r, s} u_{r} u_{s}\left(R_{r s}^{\prime}-\sum_{i, \mu} B_{r i}^{\mu} B_{s i}^{\mu}\right)\right\} * 1 .
\end{aligned}
$$

TheOREM 2. Let $N$ be an $n$-dimensional submanifold in $S^{n+p}$ and $B$ the second fundamental form of $N$. If $\|B\|^{2}<(n-1) K$, where $K(x)$ denotes the infimum of the sectional curvature of $N$ at the point $x$, then there is no nonconstant stable minimal submanifold in $N$. 
Proof. Since $L$ is a vector space of finite dimension, we can compute the trace of $\tilde{I}_{f}$ over $L$. In a similar way as in Theorem 1 , we have

$$
\operatorname{trace} \tilde{I}_{f}=-\int_{M}\left\{\sum_{r} R_{r r}^{\prime}-\sum_{i, r, \mu}\left(B_{r i}^{\mu}\right)^{2}\right\} * 1,
$$

where $R_{r s}^{\prime}$ is defined by (1.5). Our hypotheses give

$$
\sum_{r} R_{r r}^{\prime}=\sum_{r, i} R_{r i r i}^{\prime} \geqslant m(n-m) K \geqslant(n-1) K,
$$

where $K(x)$ denotes the infimum of the sectional curvature of $N$ at the point $x$, and

$$
\sum_{i, r, \mu}\left(B_{r i}^{\mu}\right)^{2} \leqslant \sum_{\mu, \alpha, \beta}\left(B_{\alpha \beta}^{\mu}\right)^{2}=\|B\|^{2}
$$

Hence

$$
\text { trace } \tilde{I}_{f} \leqslant \int_{M}\left\{\|B\|^{2}-(n-1) K\right\} * 1
$$

Now the conclusion is obvious.

REMARK 2. When $\|B\|=0, N$ is just the Euclidean sphere $S^{n}$ and this theorem becomes Simons' theorem.

REMARK 3. It is obvious from the proof that the same conclusion holds when the ambient space $S^{n+p}$ is replaced by the Euclidean space $R^{n+p}$. In particular, we have

THEOREM 3. If $N$ is a convex hypersurface in the Euclidean space $R^{n+1}$, there exists no $m(<n)$-dimensional stable compact minimal submanifold $M$ in $N$ such that at each point of $M$ the tangent space of $M$ is exactly spanned by $m$ principal directions of $N$.

Proof. In this case we choose at each point the principal directions of $N$ in computing the trace of $\tilde{I}_{f}$ over $L$. Thus, since $p=1$, and using the convexity of $N$, (2.11) becomes

$$
\operatorname{trace} \tilde{I}_{f}=-\int_{M}\left(\sum_{r} R_{r r}^{\prime}\right) * 1<0
$$

The proof is complete.

REMARK 4. Since $S^{n}$ is an umbilical convex hypersurface in $R^{n+1}$, this theorem is also a generalization of Simons' theorem in another direction.

EXAMPLE. Consider the torus $T^{2} \hookrightarrow R^{3}$. Since the greatest latitude belongs to a part of $T^{2}$, which itself is a convex hypersurface in $R^{3}$, it is obvious from Theorem 3 that the greatest latitude circle of $T^{2}$ is not a stable geodesic.

3. Other results. In the following, we study the relation between the stability of a minimal immersion and the stability of a harmonic map.

Let $f: M \rightarrow N$ be a minimal immersion. Then its index from $\tilde{I}_{f}$ is expressed by (1.2). On the other hand, when we consider $f$ as a harmonic map, the index form $I_{f}$ is 
expressed by (1.1). But, in this case, we have $a_{\alpha i}=\delta_{\alpha i}$ and therefore

$$
I_{f}(V, V)=-\int_{M}\left\{\sum_{\alpha} v_{\alpha}\left(\Delta v_{\alpha}+\sum_{\beta} v_{\beta} R_{\alpha \beta}^{\prime}\right)\right\} * 1 .
$$

When the deformation vector is normal to $f(M)$, i.e., $v_{i}=0$, we have, by a straightforward computation,

$$
I_{f}(V, V)=\tilde{I}_{f}(V, V)+2 q(V, V),
$$

where $q(V, V)$ is a positive semidefinite quadratic form defined by

$$
q(V, V)=\int_{M} \sum_{r, s} Q_{r s} v_{r} v_{s} * 1=\int_{M}\left\{\sum_{i, j}\left(\sum_{r} a_{r i j} v_{r}\right)^{2}\right\} * 1
$$

From (3.2) we easily obtain

THEOREM 4. Let $f: M \rightarrow N$ be a minimal immersion and $I_{f}$ the index form of $f$ as a harmonic map. Then with respect to any deformation vector normal to $f(M), I_{f}=\tilde{I}_{f}$ if and only if the immersion $f$ is totally geodesic.

COROLlaRY 1. Let $f: M \rightarrow N$ be a minimal immersion. If $f$, as a harmonic map, is unstable with respect to the normal variation of $f(M)$, then $f(M)$ is an unstable minimal submanifold in $N$.

COROLlaRY 2. If $f: M \rightarrow N$ is a totally geodesic immersion, then $f$ is stable as a harmonic map if and only if $f$ is stable as a minimal immersion.

ACKNOWLEDGEMENT. The first-named author would like to thank the MPI für Mathematik and the SFB 40 of the University of Bonn for their hospitality, and the authors would also like to thank the referee for his helpful suggestions for improving the first version.

\section{REFERENCES}

1. S. S. Chern, Minimal submanifolds in Riemannian manifolds, Mimeographed Lecture Notes, Univ. of Kansas, 1968.

2. S. S. Chern and S. I. Goldberg, On the volume decreasing property of a class of real harmonic mappings, Amer. J. Math. 97 (1975), 133-147.

3. J. Eells and J. H. Sampson, Harmonic mappings of Riemannian manifolds, Amer. J. Math. 86 (1964), 109-160.

4. P. F. Leung, On the stability of harmonic maps, Lecture Notes in Math., vol. 949, Springer, New York and Berlin, 1982, pp. 122-129.

5. M. Obata, The Gauss map of immersions of Riemannian manifolds in a space of constant curvature, J. Differential Geom. 2 (1968), 217-223.

6. Y. L. Pan, Some nonexistence theorems on stable harmonic mappings, Chinese Ann. Math. 3 (1982), $515-518$.

7. Y. L. Pan and Y. B. Shen, Stable harmonic maps from submanifolds in space form, preprint.

8. C. K. Peng, Some relations between the minimal submanifold and the harmonic mapping, Chinese Ann. Math. Ser. A 5 (1984), 85-89.

9. R. T. Smith, The second variation formula for harmonic mappings, Proc. Amer. Math. Soc. 47 (1975), 229-236.

10. J. Simons, Minimal varieties in Riemannian manifolds, Ann. of Math. (2) 88 (1968), 62-105. 
11. Y. L. Xin, Topology of certain submanifolds in the Euclidean sphere, Proc. Amer. Math. Soc. 82 (1981), 643-648.

12. __ Some results on stable harmonic maps, Duke Math. J. 47 (1980), 609-613.

MAX - PlanCK - INSTITUT FUR MATHEMATIK UND SONDERFORSCHUNGBEREICH 40 DER UNIVERSITÄT BonN, Federal Republic of Germany

Department of Mathematics, Fudan University, Shanghai, People's Republic of China (Current address of Y. L. Pan)

Department of Mathematics, Hangzhou University, Hangzhou, People's Republic of China (Current address of Y. B. Shen) 